\title{
Nasal obstruction and smell impairment in nasal polyp disease: correlation between objective and subjective parameters*
}

\author{
V. Hox ${ }^{1,2}$, I. Callebaut ${ }^{1}$, S. Bobic ${ }^{1}$, M. Jorissen ${ }^{2}$, P.W. Hellings ${ }^{1,2}$ \\ 1 Laboratory of Experimental Immunology, University Hospitals Leuven, Catholic University of Leuven, \\ Leuven, Belgium \\ 2 Department of Otorhinolaryngology, Head and Neck Surgery, University Hospitals, Leuven, Catholic \\ University of Leuven, Leuven, Belgium
}

\begin{abstract}
SUMMARY
Background: Background: Chronic rhinosinusitis with nasal polyposis (NP) represents an invalidating disorder that causes mainly nasal blockage and loss of smell. The aim of this study is to investigate correlations between individual subjective and objective parameters of stable NP disease.

Methods: Sixty five NP patients scored their sinonasal symptoms on a visual analogue scale (VAS) and questionnaires (SNOT-22 and SF-36). Peak nasal inspiratory flow (PNIF) measurement, Sniffin' Sticks (SS) smell test, blood analysis for eosinophilia, total IgE and culture for Staphylococcus aureus (SA) were performed.

Results: VAS scores for nasal blockage correlated with the SNOT-22 and SF-36 scores, which was not observed for VAS of other symptoms. VAS scores for nasal blockage correlated well with PNIF values as well as VAS scores for smell dysfunction and SS results. NP size correlated with VAS scores for nasal blockage, but not with VAS for other symptoms. NP size showed an inverse correlation with PNIF and SS scores. Blood eosinophilia correlated with subjective smell reduction. The presence of $S A$ or total IgE levels were not associated with symptoms of NP disease.

Conclusion: PNIF and SS are good tools to evaluate symptoms of nasal obstruction and smell reduction in NP disease. Nasal blockage is the only symptom that correlates well with NP size and SNOT-22 scores, whereas smell reduction correlates with blood eosinophilia.
\end{abstract}

Key words: nasal polyps, nasal obstruction, smell, visual analogue scale, correlations

\section{INTRODUCTION}

Chronic rhinosinusitis (CRS) with Nasal Polyps (NP) is defined as an inflammatory condition of the nose and paranasal sinuses characterized by sino-nasal symptoms and the presence of polyps inside both nasal cavities ${ }^{(1)}$. Symptoms related to NP are mainly nasal obstruction and loss of smell, both having a severe impact on the quality of life of affected patients ${ }^{(2)}$. The alleviation of these symptoms by medical and/or surgical treatment is the ultimate goal of any therapeutic regimen.

The scope of this observational study is to investigate the correlation between the two primary symptoms of NP disease, i.e. nasal obstruction and smell disorder, and objective parameters of the disease. In clinical practice, several instruments are available to evaluate the presence and severity of disease. Nasal endoscopy is used to diagnose and evaluate the size of NP and response to treatment ${ }^{(3)}$. Several techniques can be used for the objective evaluation of nasal flow and patency: acoustic rhinometry (AR), anterior or posterior rhinomanometry (RM) and Peak Nasal Inspiratory Flow (PNIF) ${ }^{(4,5)}$. Several smell tests are commercially available to evaluate the patients' smell capacity, like the UPSIT ${ }^{\left({ }^{6}\right)}$, Sniffin' Sticks ${ }^{(7)}$, Zurich Smell Diskette ${ }^{(8)}$ and the Barcelona smell test ${ }^{(9)}$. Every single technique for evaluation of nasal patency and smell capacity has its' specific advantages and disadvantages, related to the costs, technical requirements, duration of procedure and reliability of generated data. Ideally a rapid, budget-friendly and reliable technique should be advocated in routine clinic for objective evaluation of the nasal symptoms. Beside impairment of nasal patency and smell capacity, NP disease is frequently associated with increased blood eosinophilia and IgE in the serum and local immune response to Staphylococcus aureus enterotoxins (SAE), with IL-5 and specific IgEs directed against SAE in the NP tissue ${ }^{(10)}$. 
So far, little is known about the relationship between the cardinal symptoms of NP and clinically relevant objective parameters. Previously, two observational studies described a moderate to good correlation between subjective nasal obstruction and results of PNIF measurements in healthy or allergic patients ${ }^{(11,12)}$. Alobid et al. ${ }^{(13)}$ reported previously in nasal polyp patients that there exists no correlation between quality of life and the scoring of nasal symptoms, polyp size, nasal patency, nasal and blood eosinophilia and serum total IgE. To our knowledge, no other data are available on correlations between nasal symptom scoring and technical measurements. The same holds true for the relationship between the extent of $\mathrm{NP}$ and subjective symptom scoring on the one hand and the outcomes of objective tests on the other hand ${ }^{(14,15)}$. The aim of this observational study is to investigate the correlations between different parameters of NP disease that determine the major symptoms of nasal blockage and smell reduction in the patients.

\section{MATERIALS AND METHODS}

\section{Study population}

This observational cross-sectional study involved a convenience sample of 65 adult patients suffering from CRS with NP who consulted the Ear, Nose and Throat Department of the University Hospitals Leuven between September 2006 and August 2008. All the patients suffered from stable sino-nasal symptoms for more than 12 weeks and had bilateral NP disease despite the daily use of a nasal corticosteroid spray at recommended dose for more than 4 weeks (MF $200 \mu \mathrm{g}$ or FP 200 $\mu \mathrm{g}$ once daily). Patients with an infectious exacerbation of rhinosinusitis in the previous 4 weeks were excluded. Patients who underwent sinus surgery within the previous 3 months or who were treated with systemic steroids in the month prior to evaluation were excluded as well. Patients suffering from cystic fibrosis, primary ciliary dyskinesia or other systemic vasculitic or granulomatous disease were not included. The sensitization state to common inhalant allergens was evaluated by specific IgE detection in the serum or by skin prick tests (SPT). The presence of aspirin hypersensitivity and current smoking habit was based on the patients' history. The presence of lower airway symptoms corresponds to a positive history for either wheezing, dyspnea in rest or during the night.

Prior to inclusion, all patients signed an informed consent. The study was approved by the local Medical Ethical Committee of the University Hospitals Leuven.

All of the clinical investigations and tissue handling above were performed by the same clinician (P.H.) in all patients.

\section{Evaluation of sino-nasal symptoms in NP patients}

To obtain a subjective assessment of disease severity, we asked the patients to evaluate their current sino-nasal symptoms on a visual analogue scale (VAS) as described before ${ }^{(16)}$. Patients were asked to mark the severity of their symptoms on a horizontal line of 0 till $10 \mathrm{~cm}$, with 0 meaning no problem and 10 the worst thinkable problem. The following symptoms were scored: nasal blockage, loss of smell, post nasal drip (PND), rhinorrhea and headache. In addition, patients were asked to fill in the Sino-Nasal Outcome Test (SNOT) 22, a disease-specific questionnaire on rhinosinusitis symptoms ${ }^{(2,17)}$. This questionnaire involves 22 symptoms associated with sino-nasal disease and patients were asked to score every item from 0 to 5 . For evaluation, the total SNOT score was divided by 22. To evaluate the impact of the sino-nasal symptoms on the quality of life (QOL), the short form (SF)-36 questionnaire was used containing 36 questions on physical health $(\mathrm{PH})$ and mental health $(\mathrm{MH}){ }^{(18)}$. All items were scored on a 0 to 100 range so that they represent the percentage of total possible score achieved.

\section{Evaluation of NP size}

NP size was scored on both sides by nasal endoscopy as follows: 0 : none, 1: cobblestone, 2: nasal polyps not reaching below the upper border of the middle nasal turbinate, 3 : nasal polyps reaching below the upper border of the middle nasal turbinate 4: nasal polyps reaching below the inferior border of the inferior nasal turbinate, 5: nasal polyps reaching the floor of the nasal cavity. This was done for both anterior and posterior ethmoid. The sum of the scores was made with a maximum score of 20 as described before ${ }^{(19)}$. Only patients with a minimum NP score of $8 / 20$ (sum of both sides) were included, which corresponds with moderate and severe NP disease.

\section{Evaluation of nasal peak flow}

Nasal flow was quantified by measuring the PNIF using the Peak Nasal Inspiratory Flow device (Cement Clarkx International, Essex, UK). Three satisfactory maximal inspirations with a variability of $<10 \%$ were obtained and the highest value was recorded ${ }^{(4)}$. Data are expressed in $\mathrm{L} / \mathrm{min}$.

\section{Evaluation of smell capacity}

Olfactory function was evaluated using a Sniffin' Sticks (SS) identification odour test ${ }^{(7)}: 12$ odours were blindly presented to the patients along with a multiple choice list of 4 responses each. Every correct odour identification was assigned one point. Data are expressed as an absolute number ranging from 0 to 12 , with 0 meaning no correct identification and 12 being the highest odour identification score.

\section{Evaluation of colonization of the nasal cavity with Staphylococcus aureus}

A swab was taken from the middle meatus of 54 patients using a cotton tipped applicator and sent for culture of Staphylococcus aureus (SA) within $24 \mathrm{~h}$. In the clinical laboratory, swabs were inoculated on a rich universal blood agar and on a mannitol salt agar and incubated overnight at $37^{\circ} \mathrm{C}$ to evaluate specific growth of SA, as reported elsewhere ${ }^{(20)}$. 
Table 1. Patients' characteristics.

\begin{tabular}{lc}
\hline Variables & $\mathrm{n}(\mathrm{tot}=65)$ \\
\hline Age (mean \pm SD in yrs) & $51.7 \pm 13.3$ \\
Gender: male (\%) & $55(85)$ \\
Current smokers (\%) & $9(14)$ \\
Allergy to common allergens (\%) & $21(32)$ \\
Lower airway symptoms (\%) & $21(32)$ \\
Aspirin hypersensitivity (\%) & $11(17)$ \\
Colonization with Staphylococcus aureus (\%) & $20(37)$ \\
\hline
\end{tabular}
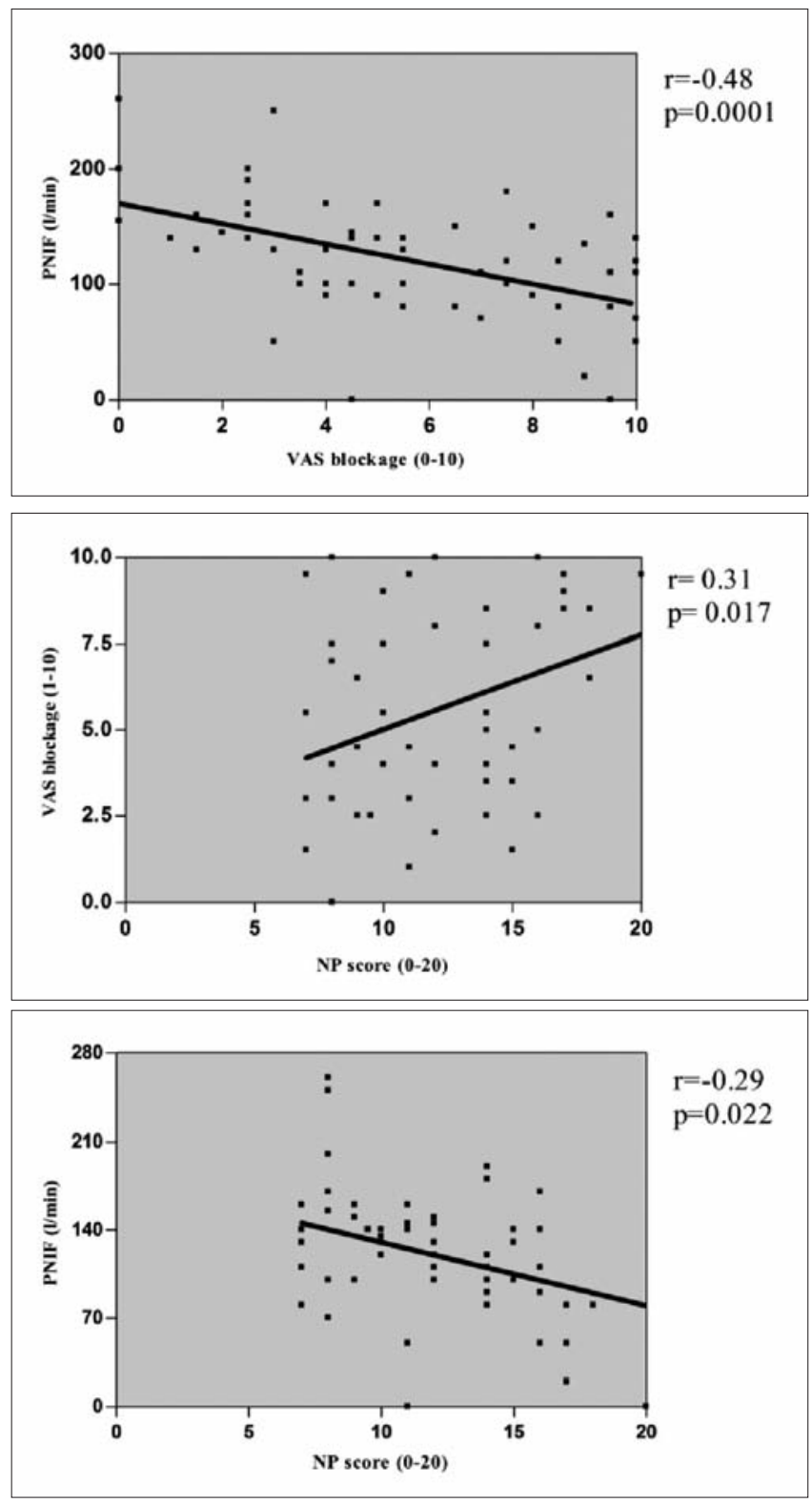

Figure 1. Nasal Obstruction. Linear regression graphs for objective and subjective measurements of nasal obstruction: a) VAS score for nasal blockage and results of PNIF measurements $(r=-0.48, p=0.0001)$; $b)$ nasal polyp (NP) scoring and VAS score for nasal blockage $(r=0.31$, $p$ $=0.017)$; $\mathrm{c}$ ) nasal polyp (NP) scoring and results of PNIF measurements $(r=-0.29, p=0.022)$.
Evaluation of blood eosinophilia and total IgE

A venipuncture was performed and blood was analyzed for systemic eosinophilia and total IgE using the ImmunoCap ${ }^{\circledR}$ system.

\section{Statistical analyses}

Correlations between the different variables were analyzed using linear regression and the Spearman coefficient was calculated to evaluate the degree of correlation in a non-parametric population. A correlation was considered to be significant if $\mathrm{p}<0.05$ (two-tailed, CI of 95\%). Comparisons between two variables were calculated using a Mann-Whitney test.

\section{RESULTS}

\section{Patient characteristics}

The patient characteristics are summarized in Table 1. The mean age of the patients was $51.7 \mathrm{yr}(25-82)$ and there was a male preponderance of $85 \%$. About one third of the patients $(32 \%)$ was allergic to one or more common aero-allergen and $14 \%$ were current smokers. Of the NP patients, $37 \%$ were colonized with Staphylococcus aureus (SA).

Nasal obstruction and its' correlation with NP disease parameters Correlations between the different parameters that determine nasal obstruction were measured. Nasal obstruction evaluated using VAS scores correlated inversely with PNIF values $(r=-0.48, p=0.0001$, Figure 1a) and it showed a positive correlation with NP size evaluated with nasal endoscopy $(r=0.31$, $\mathrm{p}<0.05$, Figure $1 b$ ). The negative correlation between NP size and PNIF measurements was significant as well with $r=-0.29$ $(p<0.05$, Figure 1c). VAS scores for nasal obstruction correlated with the results of the SNOT-22 questionnaire $(r=0.42$, $\mathrm{p}<0.01$, Table 2). On the other hand, correlations between results of SF36 questionnaire and the VAS for nasal blockage failed to reach statistical significance.

Correlations between VAS scores for nasal blockage and other objective parameters of NP disease like blood eosinophilia and total peripheral $\mathrm{IgE}$ were not statistically significant (Table 2). When VAS scores for nasal blockage were compared between asthmatics and non-asthmatics, between aspirin sensitive and non-sensitive patients and between smokers and non-smokers, there was no significant difference (data not shown).

\section{Smell dysfunction and its' correlation with disease parameters}

We found a strong negative correlation between the subjective VAS scores for loss of smell and results of the objective Sniffin' Sticks test $(r=-0.72, p<0.0001$, Figure 2a). However, there was no significant correlation between the VAS scores for smell reduction and the NP size (Figure 2b). An inverse correlation between the NP size and SS scores was found $(\mathrm{r}=-0.38, \mathrm{p}<0.01$, Figure $2 \mathrm{c}$ ). There existed no significant correlations between VAS scores for smell reduction and results from the SNOT-22 or SF-36 questionnaire (Table 2). We could not detect a significant difference in VAS scoring for 

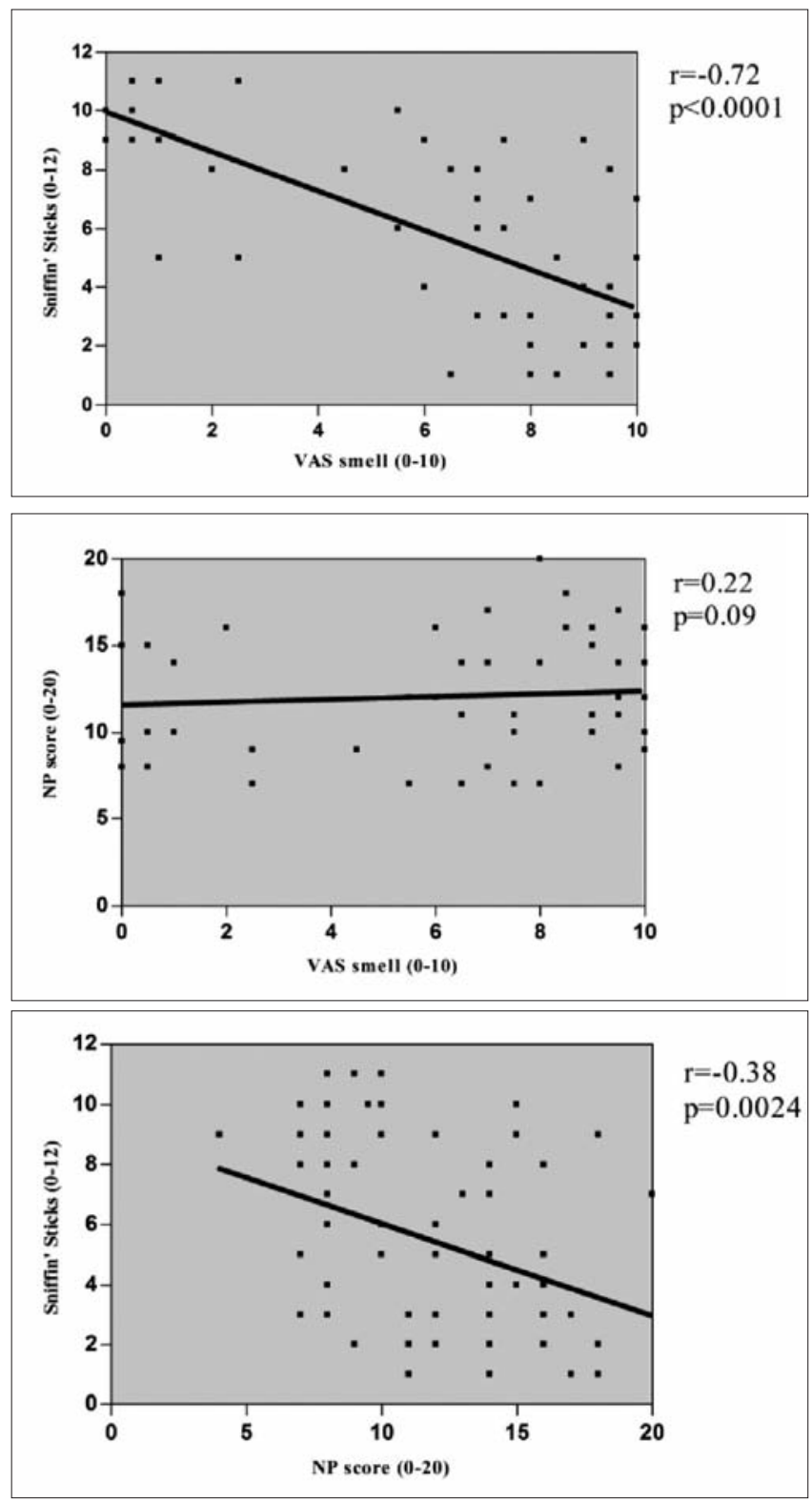

Figure 2. Smell Reduction. Linear regression graphs for objective and subjective measurements of smell reduction: a) VAS score for loss of smell and results of Sniffin' Sticks $(r=-0.72, p<0.0001)$; $b)$ nasal polyp (NP) scoring and VAS score for loss of smell $(\mathrm{r}=0.22, \mathrm{p}=0.09)$; c) nasal polyp (NP) scoring and results of Sniffin' Sticks ( $\mathrm{r}=-0.38, \mathrm{p}=$ 0.0024).

smell reduction between asthmatics and non-asthmatics, aspirin sensitive and non-sensitive patients or between smokers and non-smokers (data not shown).

\section{The significance of blood eosinophilia in NP disease}

The correlation between VAS scores for loss of smell and blood eosinophilia was significant $(r=0.45, \mathrm{p}<0.001$, Figure $3 a)$. The inverse correlation between the objective SS test and blood eosinophilia was significant as well $(r=-033, p=0.01$, Figure $3 \mathrm{~b}$ ). In contrast, blood eosinophilia did not significantly correlate with VAS scores for nasal blockage $(r=0.097, p>$
$0.05)$ or PNIF outcomes $(r=0.02, p>0.05)$. When NP size was compared to blood eosinophilia, only a weak correlation $(\mathrm{r}=0.16)$ was found without reaching significance. No other significant correlations existed between blood eosinophilia and other subjective and objective parameters of nasal polyp disease (Table 2).

\section{The significance of colonization with SA in NP disease}

When different parameters of NP disease were evaluated in relation to colonization with SA, we did not find a significant difference between the patient groups. Neither the VAS scores for individual symptoms, nor results of SNOT-22 nor SF-36 questionnaires differed between SA positive patients and SA negative patients. The same observation was made when NP size was compared between the two groups. Results of objective parameters like nasal patency, smell tests and blood tests did not differ significantly either between patients colonized with SA and patients not colonized (data not shown).

\section{DISCUSSION}

The diagnosis of CRS with NP is made based on the combination of symptoms mentioned by the patient and objectification of disease parameters by the clinician using nasal endoscopy or CT scan imaging ${ }^{(1)}$. Additional tests like evaluation of nasal patency smell tests, blood eosinophil count and IgE titer, may be applied to better characterize and evaluate the extent and
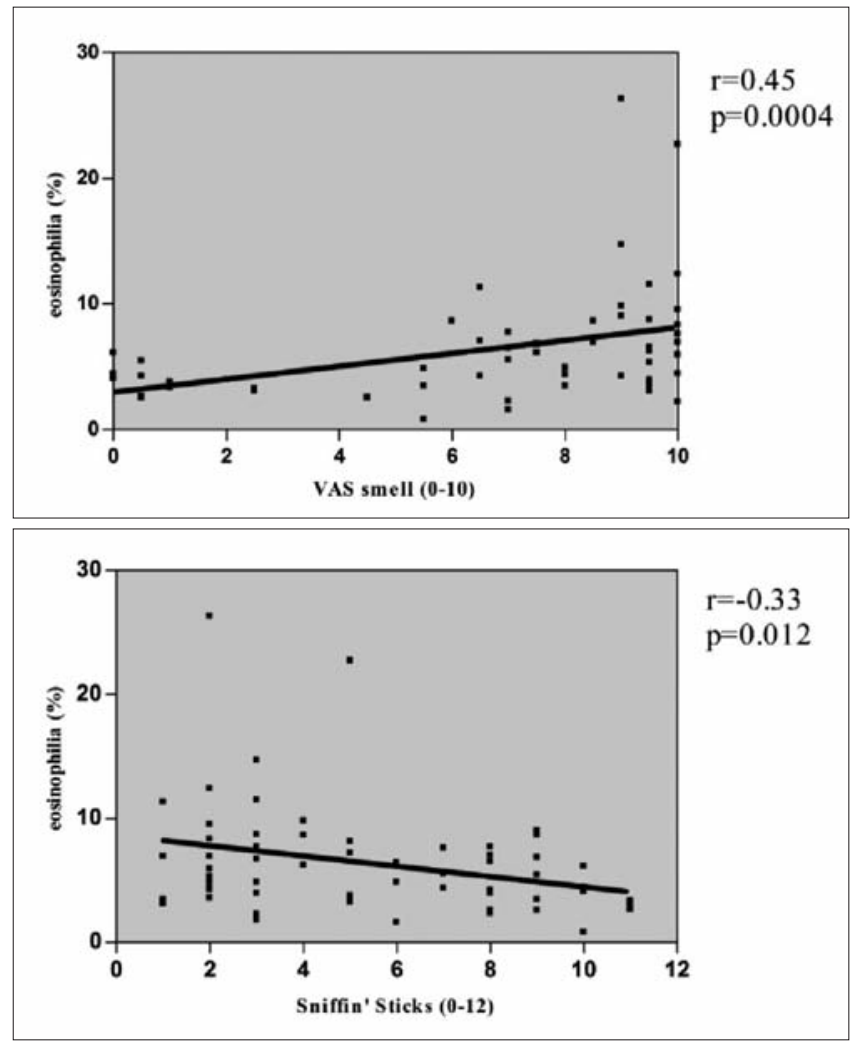

Figure 3. Blood Eosinophilia. Linear regression graphs for blood eosinophilia and a) VAS score for loss of smell $(\mathrm{r}=0.45, \mathrm{p}=0.0004)$, b) results of Sniffin' Sticks smell test $(r=-0,33, p=0.012)$. 
Table 2. Correlations between different NP parameters.

\begin{tabular}{|c|c|c|c|c|c|c|c|c|c|}
\hline & $\begin{array}{c}\text { Spearman } \\
\text { p-value }\end{array}$ & & & & & Coefficien & & & (r) \\
\hline & $V A S$ & $V A S$ & PNIF & $S S$ & $N P$ & Eosino & Total & SNOT & $S F$ \\
\hline & block & smell & & & score & & $\operatorname{Ig} E$ & 22 & 36 \\
\hline$V A S$ & 1.0000 & 0.0729 & -0.479 & -0.07 & 0.3147 & 0.0974 & 0.002 & 0.4177 & -0.264 \\
\hline blockage & & 0.5866 & 0.0001 & 0.6039 & 0.0171 & 0.4792 & 0.9883 & 0.0011 & 0.0511 \\
\hline VAS smell & 0.0729 & 1.00000 & 0.1736 & -0.720 & 0.2219 & 0.4468 & 0.0298 & 0.1198 & 0.1480 \\
\hline & 0.5866 & & 0.1808 & $<.0001$ & 0.0883 & 0.0004 & 0.8191 & 0.3619 & 0.2675 \\
\hline PNIF & -0.479 & 0.17363 & 1.0000 & -0.01 & -0.294 & 0.0232 & 0.1249 & -0.210 & 0.2448 \\
\hline & 0.0001 & 0.1808 & & 0.9396 & 0.0216 & 0.8618 & 0.3334 & 0.1043 & 0.0616 \\
\hline Sniffin' & -0.07 & -0.7206 & -0.01 & 10000 & -0.382 & -0.325 & 0.0319 & -0.089 & -0.078 \\
\hline Sticks (SS) & 0.6039 & $<.0001$ & 0.9396 & & 0.0024 & 0.0120 & 0.8056 & 0.4949 & 0.5585 \\
\hline Nasal Polyp & 0.3147 & 0.22193 & -0.294 & -0.382 & 1.0000 & 0.1564 & -0.048 & 0.0322 & 0.1060 \\
\hline$(N P)$ score & 0.0171 & 0.0883 & 0.0216 & 0.0024 & & 0.2409 & 0.7124 & 0.8068 & 0.4284 \\
\hline Blood & 0.0974 & 0.44683 & 0.0232 & -0.325 & 0.1564 & 1.0000 & 0.2405 & 0.1762 & -0.185 \\
\hline Eosinophilia & 0.4792 & 0.0004 & 0.8618 & 0.0120 & 0.2409 & & 0.0665 & 0.1858 & 0.1717 \\
\hline Total IgE & 0.002 & 0.02989 & 0.1249 & 0.0319 & -0.048 & 0.2405 & 10000 & 0.0331 & -0.071 \\
\hline & 0.9883 & 0.8191 & 0.3334 & 0.8056 & 0.7124 & 0.0665 & & 0.7999 & 0.5923 \\
\hline SF 36 & -0.264 & 0.14800 & 0.2448 & -0.078 & 0.1060 & -0.185 & -0.071 & -0.507 & 1.0000 \\
\hline & 0.0511 & 0.2675 & 0.0616 & 0.5585 & 0.4284 & 0.1717 & 0.5923 & $<.0001$ & \\
\hline
\end{tabular}

Correlations between the investigated parameters in polyp patients. A negative Spearman Coefficient means that there is an inverse correlation. Below the Spearman Coefficient the p-values of the correlations are noted. (VAS: visual analogue scale, PNIF: peak nasal inspiratory flow, SNOT: sino-nasal outcome test, SF-36: short form-36).

severity of the disease as well as to objectify the effects of any medical or surgical treatment. So far, little is known about the relationship between the subjective symptom perception and the different objective measurements of NP disease. The major symptoms of NP disease are nasal obstruction and loss of smell and different techniques exist to evaluate these symptoms ${ }^{(1)}$.

The most important symptom of NP disease is nasal obstruction, which can be objectively evaluated by rhinomanometry (RM), acoustic rhinometry (AR) and PNIF among other less frequently used tools. Some studies have shown a good inverse correlation between outcomes of RM, and especially $\mathrm{AR}$ and the subjective sensation of nasal blockage in rhinitis or rhinosinusitis patients ${ }^{(21-23)}$. Since these techniques are not routinely used in daily practice, we chose the PNIF device to evaluate nasal obstruction in NP patients, which has been shown to correlate well with subjective nasal obstruction ${ }^{(11,12)}$. Here a significant inverse correlation between PNIF results and subjective sensation of nasal blockage was confirmed. So far no study investigated the correlation between PNIF outcome and NP size, although Lund et al. showed an increase in PNIF after reduction in NP size by topical steroids ${ }^{(24)}$. In this study, we found a significant inverse correlation, although less strong, between the PNIF measurement and the endoscopic score of NPs in the patients. Therefore, our data illustrate that PNIF can be a useful tool in evaluating the nasal obstruction in NP disease in clinical practice. However, the fact that a high inter-individual variability is found (Figure 1a) ${ }^{(4)}$ limits the conclusions from a single measurement in one individual and therefore remains a disadvantage of the technique. Despite this fact, PNIF measurement represents a valuable tool to evaluate changes in flow in response to treatment and comparing different patient groups. We also showed a moderate correlation between the VAS scores for nasal blockage and the results of the SNOT-22 questionnaire. This questionnaire is a modification of the validated disease specific SNOT-20 questionnaire and comprises questions about nasal obstruction and loss of smell and taste ${ }^{(2)}$. However, results of the SNOT-22 questionnaire did not correlate with any of the other VAS scores of NP symptoms. No significant correlations were found between results of the SF-36 questionnaire on quality of life in general and the VAS scoring of NP patients.

Beside nasal obstruction, smell dysfunction represents a cardinal symptom of NP disease: the median VAS score for smell reduction of all the included NP patients was 6,5. Smell dysfunction can be evaluated by several standardized smell tests. 
In this study we chose the Sniffin' Sticks, because it is a standardized method for objective assessment of smell reduction allowing a rapid evaluation of smell capacity ${ }^{(7)}$. Moreover, it is a user-friendly and relatively cheap technique. We found a strong and significant inverse correlation with the VAS scoring for loss of smell. Interestingly, a moderate but significant inverse correlation was found between the results of the smell test and the extent of NP. Therefore, Sniffin' Sticks represents a good technique to evaluate the reduction of smell in NP patients clinical practice.

Our next question was whether these symptoms mentioned by NP patients correspond with the size of the polyps evaluated by nasal endoscopy. Clinicians are sometimes confronted with patients presenting with giant polyps in the nasal cavities who have hardly any complaints and others with very small polyps complaining of complete anosmia or blocked nose. In this study, we found a moderate correlation between the size of the NP and VAS scores for nasal blockage, but only a very weak and non significant correlation between polyp size and VAS scores for smell reduction, rhinorrhea, headache and PND. This means that we cannot rely on NP size to make an estimation of the patients' subjective disease perception. Neither can we ground treatment strategies on the extent of NP alone.

A possible shortcoming of the measurements on this subject can be that we did not take into account the localization of the polyps, since it is probable that polyps obstructing the olfactory area cause more smell reduction than those filling only the middle and inferior meatus and vice versa for nasal obstruction.

NP disease in European patients is characterized by a Th2 type eosinophilic inflammation and Th2 related cytokines are known to be up regulated in NP tissue compared to controls ${ }^{(20)}$. Therefore, we checked eosinophil count in the blood. It was surprising to find that both the subjective and objective loss of smell significantly correlated with blood eosinophilia. This is indeed consistent with the findings of Haruna et al., who found higher VAS scores for loss of smell in patients with high numbers of activated (EG2+) eosinophils in the blood ${ }^{(25)}$. A possible explanation for this finding lies in the fact that polyp patients who have the best preservation of smell are the most regular users of topical steroids, since this treatment is known to have a beneficial effect on smell ${ }^{(26)}$. The topical steroids are also known to cause a reduction in eosinophils, although this is only proven for eosinophils present in the tissue and not in the blood ${ }^{(27)}$.

Growing evidence is available on a potential involvement of Staphylococcus aureus (SA) in the pathogenesis of nasal polyp disease $^{(10)}$. There is a higher colonization rate in polyp patients that is even more prominent when there is concomitant asthma or aspirin hypersensitivity (up to $87.5 \%)^{(28)}$. $S A$ enterotoxins (SAE) are superantigens that induce polyclonal T-cell expansion with induction of IL-4 and IL-5 and in this way attract and activate eosinophils to the nasal mucosa ${ }^{(10,28)}$. Here, no significant differences were seen between positive culture for $S A$ and different subjective and objective parameters. NP size did not differ between $S A$ positive and negative patients. Neither did outcomes of VAS scores, PNIF, smell tests, questionnaires, blood IgE or eosinophilia, when compared between $S A$ positive and negative patients. The explanation for the lack of correlation between the presence of $S A$ and more severe NP disease relates to the fact that not the presence of $S A$ per se but rather the immune response to the enterotoxins of $S A$ represents an important factor in aggravation of the airway disease ${ }^{(20,29)}$.

Taken together, this study shows the usefulness of both PNIF and Sniffin' Sticks in the evaluation of respectively nasal obstruction and smell dysfunction in NP patients. Moreover, it shows that there is no strong correlation between the size of NP and subjective sensation of nasal obstruction and smell dysfunction. Finally, a positive correlation between blood eosinophilia and both subjective smell dysfunction and objective results of Sniffin' Sticks in NP patients was found.

\section{ACKNOWLEDGEMENTS}

P. Hellings is a post-doctoral research fellow and V. Hox is a research fellow of the FWO Vlaanderen.

\section{AUTHORSHIP CONTRIBUTION}

V. Hox: recruitment of patients, processing of data, writing of manuscript, I. Callebaut: aid with processing of data, S. Bobic: aid with processing of data, M. Jorissen: Head of ENT department, providing material, P.W. Hellings: Recruitment of patients, all clinical examinations/acts of/on patients

\section{CONFLICT OF INTEREST}

There exists no conflict of interest for this work.

\section{REFERENCES}

1. Fokkens W, Lund V, Mullol J. EP3OS 2007: European position paper on rhinosinusitis and nasal polyps 2007. A summary for otorhinolaryngologists. Rhinology 2007; 45: 97-101.

2. Alobid I, Bernal-Sprekelsen M, Mullol J. Chronic rhinosinusitis and nasal polyps: the role of generic and specific questionnaires on assessing its impact on patient's quality of life. Allergy 2008; 63: 1267-1279.

3. Johansson L, Akerlund A, Holmberg K, et al. Evaluation of methods for endoscopic staging of nasal polyposis. Acta Otolaryngol 2000; 120: 72-76.

4. Ottaviano G, Scadding GK, Coles S, et al. Peak nasal inspiratory flow; normal range in adult population. Rhinology 2006; 44: 32-35.

5. Malm L. Assessment and staging of nasal polyposis. Acta Otolaryngol 1997; 117: 465-467.

6. Doty RL, Shaman P, Kimmelman CP, et al. University of Pennsylvania Smell Identification Test: a rapid quantitative olfactory function test for the clinic. Laryngoscope 1984; 94: 176-178.

7. Hummel T, Sekinger B, Wolf SR, et al. 'Sniffin' sticks': olfactory performance assessed by the combined testing of odor identification, odor discrimination and olfactory threshold. Chem Senses 1997; 22: 39-52.

8. Briner HR, Simmen D. Smell diskettes as screening test of olfaction. Rhinology 1999; 37: 145-148. 
9. Cardesin A, Alobid I, Benitez P, et al. Barcelona Smell Test - 24 (BAST-24): validation and smell characteristics in the healthy Spanish population. Rhinology 2006; 44: 83-89.

10. Bachert C, Zhang N, Patou J, et al. Role of staphylococcal superantigens in upper airway disease. Curr Opin Allergy Clin Immunol 2008; 8: 34-38.

11. Gomes DL, Camargos PA, Ibiapina CC, et al. Nasal peak inspiratory flow and clinical score in children and adolescents with allergic rhinitis. Rhinology 2008; 46: 276-280.

12. Fairley JW, Durham LH, Ell SR. Correlation of subjective sensation of nasal patency with nasal inspiratory peak flow rate. Clin Otolaryngol Allied Sci 1993; 18: 19-22.

13. Alobid I, Benitez P, Valero A, et al. The impact of atopy, sinus opacification, and nasal patency on quality of life in patients with severe nasal polyposis. Otolaryngol Head Neck Surg 2006; 134: 609-612.

14. Haruna S, Otori N, Moriyama H, et al. Endoscopic transnasal transethmosphenoidal approach for pituitary tumors: assessment of technique and postoperative findings of nasal and paranasal cavities. Auris Nasus Larynx 2007; 34: 57-63.

15. Dzaman K, Pleskacz WA, Walkanis A, et al. [Taste and smell senses estimation in patients with nasal polyps]. Otolaryngol Pol 2007; 61: 831-837.

16. Ebbens FA, Scadding GK, Badia L, et al. Amphotericin B nasal lavages: not a solution for patients with chronic rhinosinusitis. J Allergy Clin Immunol 2006; 118: 1149-1156.

17. Piccirillo JF, Merritt MG, Jr., Richards ML. Psychometric and clinimetric validity of the 20-Item Sino-Nasal Outcome Test (SNOT-20). Otolaryngol Head Neck Surg 2002; 126: 41-47.

18. Ware JE, Jr., Sherbourne CD. The MOS 36-item short-form health survey (SF-36). I. Conceptual framework and item selection. Med Care 1992; 30: 473-483.

19. Hens G, Vanaudenaerde BM, Bullens DM, et al. Sinonasal pathology in nonallergic asthma and COPD: 'united airway disease' beyond the scope of allergy. Allergy 2008; 63: 261-267.

20. van ZT, Vaneechoutte M, Holtappels G, et al. Detection of enterotoxin DNA in Staphylococcus aureus strains obtained from the middle meatus in controls and nasal polyp patients. Am J Rhinol 2008; 22: 223-227.

21. Simola M, Malmberg H. Sensation of nasal airflow compared with nasal airway resistance in patients with rhinitis. Clin Otolaryngol Allied Sci 1997; 22: 260-262.
22. Numminen J, Ahtinen M, Huhtala H, et al. Comparison of rhinometric measurements methods in intranasal pathology. Rhinology 2003; 41: 65-68

23. Ciprandi G, Mora F, Cassano M, et al. Visual analog scale (VAS) and nasal obstruction in persistent allergic rhinitis. Otolaryngol Head Neck Surg 2009; 141: 527-529.

24. Lund VJ, Flood J, Sykes AP, et al. Effect of fluticasone in severe polyposis. Arch Otolaryngol Head Neck Surg 1998; 124: 513-518.

25. Haruna S, Otori N, Moriyama H. Olfactory dysfunction in sinusitis with infiltration of numerous activated eosinophils. Auris Nasus Larynx 2006; 33: 23-30.

26. Small CB, Stryszak P, Danzig M, et al. Onset of symptomatic effect of mometasone furoate nasal spray in the treatment of nasal polyposis. J Allergy Clin Immunol 2008; 121: 928-932.

27. Badia L, Lund V. Topical corticosteroids in nasal polyposis. Drugs 2001; 61: 573-578.

28. van Zele T, Gevaert P, Watelet JB, et al. Staphylococcus aureus colonization and $\mathrm{IgE}$ antibody formation to enterotoxins is increased in nasal polyposis. J Allergy Clin Immunol 2004; 114: 981-983.

29. Huvenne W, Callebaut I, Reekmans K, et al. Staphylococcus aureus enterotoxin B augments granulocyte migration and survival via airway epithelial cell activation. Allergy 2010; 65: 1013-1020.

Peter W. Hellings, M.D. Ph.D.

Department of Otorhinolaryngology, Head and Neck Surgery

University Hospitals Leuven

Kapucijnenvoer 33, 3000

Leuven

Belgium

Tel: +32-16-332 338

Fax: +32-16-346 035

E-mail: peter.hellings@med.kuleuven.be 